Journal of Innovative Optical Health Sciences

Vol. 8, No. 3 (2015) 1541008 (8 pages)

(c) The Authors

DOI: $10.1142 / \mathrm{S} 1793545815410084$

\title{
System supporting behavioral therapy for children with autism
}

\author{
Małgorzata Jędrzejewska-Szczerska*, Katarzyna Karpienko ${ }^{\dagger+}$ \\ and Agnieszka Landowska ${ }^{\dagger}$ \\ *Department of Metrology and Optoelectronics \\ Faculty of Electronics, Telecommunications and Informatics \\ Gdansk University of Technology \\ Gabriela Narutowicza Street 11/12, 80-233 Gdańsk, Poland \\ ${ }^{\dagger}$ Department of Software Engineering, Faculty of Electronics \\ Telecommunications and Informatics, Gdansk University of Technology \\ Gabriela Narutowicza Street 11/12, 80-233 Gdańsk, Poland \\ *k.karpienko@pro.wp.pl
}

Received 14 October 2014

Accepted 8 December 2014

Published 7 January 2015

\begin{abstract}
In this paper, a system supporting behavioral therapy for autistic children is presented. The system consists of sensors network, base station and a brooch indicating person's emotional states. The system can be used to measure values of physiological parameters that are associated with changes in the emotional state. In the future, it can be useful to inform the autistic child and the therapist about the emotional state of the interlocutor objectively, on the basis of performed measurements. The selected physiological parameters were chosen during the experiment which was designed and conducted by authors. In this experiment, a group of volunteers under controlled conditions was exposed to a stressful situation caused by the picture or sound. For each of the volunteers, a set of physiological parameters, was recorded, including: skin conductance, heart rate, peripheral temperature, respiration rate and electromyography. The bio-statistical analysis allowed us to discern the proper physiological parameters that are most associated to changes due to emotional state of a patient, such as: skin conductance, temperatures and respiration rate. This allowed us to design electronic sensors network for supporting behavioral therapy for children with autism.
\end{abstract}

Keywords: Autism spectrum disorder; biomedical sensors; physiological parameters; sensor network.

\$Corresponding author.

This is an Open Access article published by World Scientific Publishing Company. It is distributed under the terms of the Creative Commons Attribution 3.0 (CC-BY) License. Further distribution of this work is permitted, provided the original work is properly cited. 


\section{Introduction}

Autism spectrum disorder (ASD) causes a variety of symptoms which fall into three major categories:

(1) social interactions;

(2) verbal and nonverbal communication;

(3) repetitive and stereotyped patterns of behaviors, interests and activities. ${ }^{1}$

Impairment of social interactions, verbal and nonverbal communication, and difficulties in development and maintenance of interpersonal relationships are the characteristic traits of ASD. ${ }^{2}$

Understanding and participating in social interactions, as well as developing and maintaining positive peer relationships, are very difficult if not impossible for children with ASD. Thus, they have problems with learning, and often become easy victims of school crime and bullying. ${ }^{2,3}$

The number of children with ASD is increasing year by year. Yet still, there is a lack of effective treatment for such children. While psychopharmacology can be used as a treatment in ASD, numerous studies report the use of behavioral interventions as a more effective treatment for the basic symptoms of autism in children. According to the guidelines of National Institute for Health and Clinical Excellence, UK (NICE), a psychopharmacological treatment of challenging behaviors in individuals with autism should only be used in conjunction with non-pharmacological approaches such as behavioral therapy. ${ }^{4}$ Behavioral therapy is still the most effective way of ASD treatment. However, this kind of treatment is significantly impeded or even impossible in some cases, because of the impaired communication between the therapist and the autistic child. In this paper, we describe the system designed to help to improve behavioral therapy for autistic children by showing them the emotions of the interlocutor.

\section{Experiment}

\subsection{Experiment design}

To verify the research hypothesis, which states that it is possible to monitor human emotions using physiological signals, an experiment was designed and conducted. In the experiment, a set of physiological characteristics was measured and verified for correlation with emotional state. A range of physiological parameters that are most commonly used in emotion recognition, including: heart rate, skin conductance, respiration rate, muscle activity and peripheral temperature, were measured. The proper choice of measured parameters, as well as placement of the electrodes, was based on the data obtained from the literature. ${ }^{5-12}$ The heart rate can be obtained from electrocardiographic sensor, placed on chest, or from blood-volume pulse sensor, placed on finger tips or ears. ${ }^{5-7}$ In the experiment, blood volume pulse (BVP) was recorded in two locations simultaneously (ears and fingers) in order to immune to movement artifacts. Electromyography sensors in emotion recognition are placed on facial muscles to track microexpressions and on trapezius muscle (shoulder) because it reflects stress, ${ }^{8,9}$ and the latter location was chosen for the experiment. The sensor was doubled (on left and right trapezius muscle) in order to immune to heart muscle artifacts that might cause artifacts in the trapezius muscle signal depending on body posture change. In emotion recognition, typical locations of skin conductance sensors (SC) are the base of ring and index finger or little and middle finger. However, in the literature three alternative locations were mentioned: forearm, palm and foot. ${ }^{10,12}$ Thus, in the experiment, two locations of the SC sensors were considered - forearm and finger tips. Respiration can be measured on thorax or abdomen, and both locations were tracked in the experiment in order to record the signal independent of the respiration manner. ${ }^{8,12}$ Peripheral temperature in the experiment was measured on finger tips as well as ear lobes. Schema of sensors location on the volunteer's body, as well as experimental setup, is shown in Fig. 1.

In the experiment, we used a stimuli of $1 \mathrm{~min}$ sound, which started with $1 \mathrm{kHz}$ constant sound, and was gradually silenced and finished with a shot sound. The sound was designed to be unpleasant and such impression was confirmed by the experiment participants.

\subsection{Experiment execution}

A group of 30 healthy volunteers was involved in the experiment (16 females and 14 males) with age ranging from 22 to 56 years. Full randomization of the volunteer's selection was not possible in a setup that is located in one place only, however, the group of convenience was constructed in such a way that it represented some range of possible confounding variables, such as age and gender. 


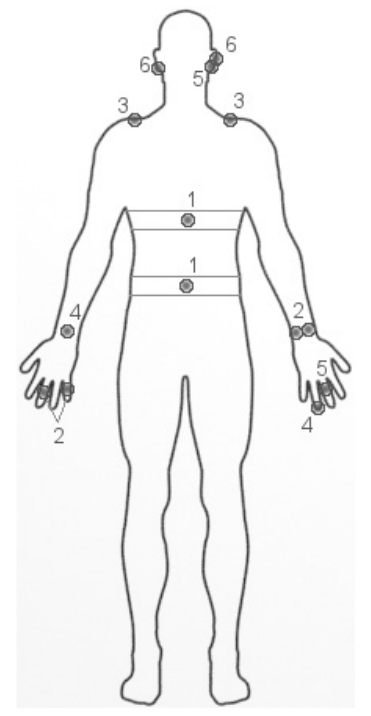

(a)

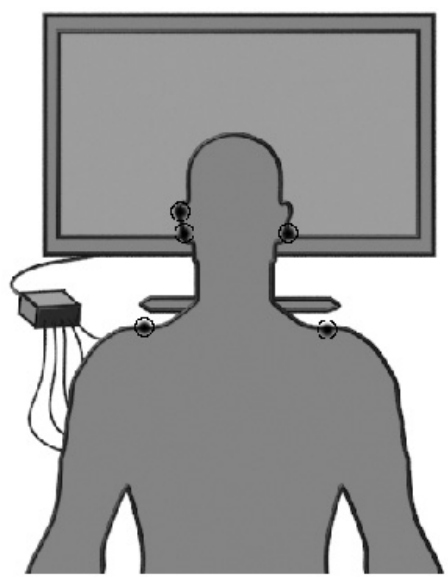

(b)

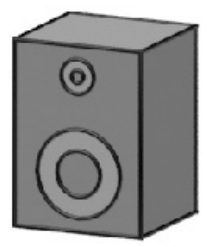

Fig. 1. (a) Location of sensors in the experiment: (1) respiration sensors; (2) SC sensors; (3) electromyography sensors; (4) temperature sensors; (5) BVP sensors; (6) electroencephalography electrodes; (b) experimental setup.
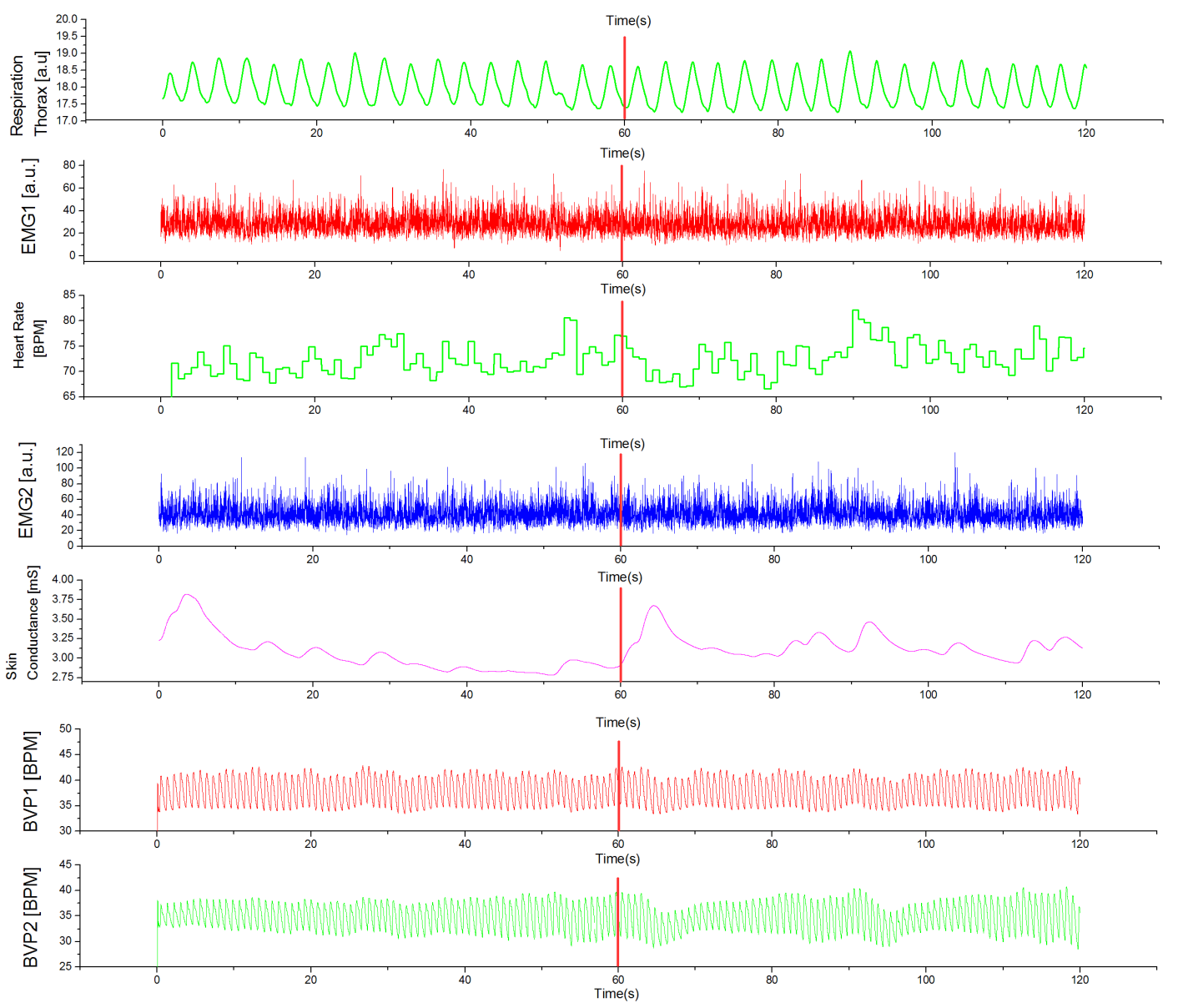

Fig. 2. An example of registered physiological parameters of one of the volunteers. Red line indicates the moment when the shot sound was fired. 
The experiment was conducted on emotion monitor stand in the laboratory at Gdańsk University of Technology. The experimental setting consists of: physiological sensors set, analytical device (FlexComp Infiniti by Thought Technology, Canada) and a computer with Thought Technology BioGraph Infiniti application for gathering biometric data from the sensors. The analytical device ${ }^{13}$ allowed making simultaneous measurement of each parameter with repetition up to $2048 \mathrm{~Hz}$.

The measurement system of physiological parameters consists of: SC sensor $(0-30.0 \mu \mathrm{S} \pm 5 \%)$, electromyography sensor $(0-2000 \mu \mathrm{V}$ RMS $\pm 5 \%)$, temperature sensor $\left(10-45^{\circ} \mathrm{C} \pm 1.0^{\circ} \mathrm{C}\right)$, as well as blood-volume pulse and respiration sensors that use relative percentage measure $(1-100 \% \pm 5 \%)$. Figure 2 shows registered physiological parameters of one of the volunteers.

Only few parameters gave very strong change in measured signal after the shout sound. In Fig. 2, it can be noted that the reaction to sound stimuli is visible after 60th $\mathrm{s}$ and the main changes of this particular session can be observed on SC, respiration and electromyography, however the individual reaction differs for each volunteer.

\section{Results}

Analysis of the results allowed us to choose which of the physiological parameters should be recorded and analyzed in a system supporting behavioral therapy for children with autism. ${ }^{14,15}$ Differences between values of reference measurement (before the shot sound) and actual measurement (after the shot sound) of each parameter are shown in Fig. 3. in the form of a box plot. Figure 3 also presents basic statistics of the results.

In Fig. 3, the main statistics of each measured parameters were estimated. The median was calculated according to the formula (1):

$$
m=\frac{x_{\frac{n}{2}}+x_{\frac{n}{2}+1}}{2},
$$

where $m$ is the median of measured values, $x$ are measured values, $n$ is the number of measured values. This formula can be used when the number of observations $n$ is an even number.

The mean was determined from Eq. (2):

$$
\bar{x}=\frac{\sum x_{i}}{n},
$$

where $\bar{x}$ is the mean of measured values, $x_{i}$ are measured values, $n$ is the number of measured values.

The interquartile range was plotted to determine the difference between the third and first quartile. Between these quartiles $50 \%$ of the results is located. Consequently, the larger interquartile range, the greater the scatter of results. It can be noted that BVP and heart rate is characterized by the biggest interquartile range. Therefore, on the basis of this statistics, these parameters should be rejected.

Standard deviation was calculated according to the formula (3) in order to give information about the physiological parameters which are characterized by the biggest scatter of the recorded changes.

$$
s=\sqrt{\frac{\sum_{i=1}^{N}\left(X_{i}-\bar{X}\right)^{2}}{n}},
$$

where $s$ is the standard deviation, $\sum_{i=1}^{N}\left(X_{i}-\bar{X}\right)^{2}$ is the sum of the squared differences between the individual results and the mean value of the population of results, $n$ is the number of obtained results.

The biggest standard deviation has been observed for BVP and heart rate. Therefore, it was once again confirmed that these parameters are too strongly dependent on the individual predispositions, in order to be used for objective determination of the emotional state of the subject. In contrast to BVP and heart rate, $\mathrm{SC}$ and respiration are characterized by relatively small interquartile range and standard deviation.

As a result of the analysis, we concluded that there is a statistically significant correlation between two physiological parameters (SC and respiration) and changes in the emotional state of individuals caused by a shot sound. The correlation between the occurrence of a stimulus and an increase of the SC is related to the galvanic skin response (GSR). GSR mechanism is based on the fact that the brain, in response to a stimulus, activates the sweat glands to secrete sweat. Perspiration causes increase of skin conductivity and consequently decrease of resistance. ${ }^{16,17}$

Change in respiration occurs due to hyperventilation. It is a condition where the number of performed inhalations and exhalations is greater than that required to maintain normal levels of oxygen in the body. ${ }^{18}$ There are substantial indications that 


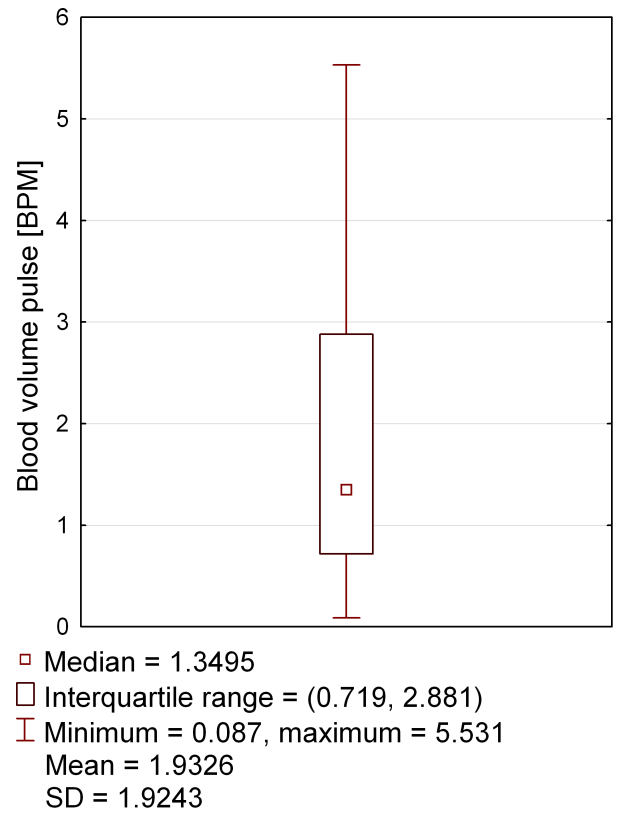

(a)

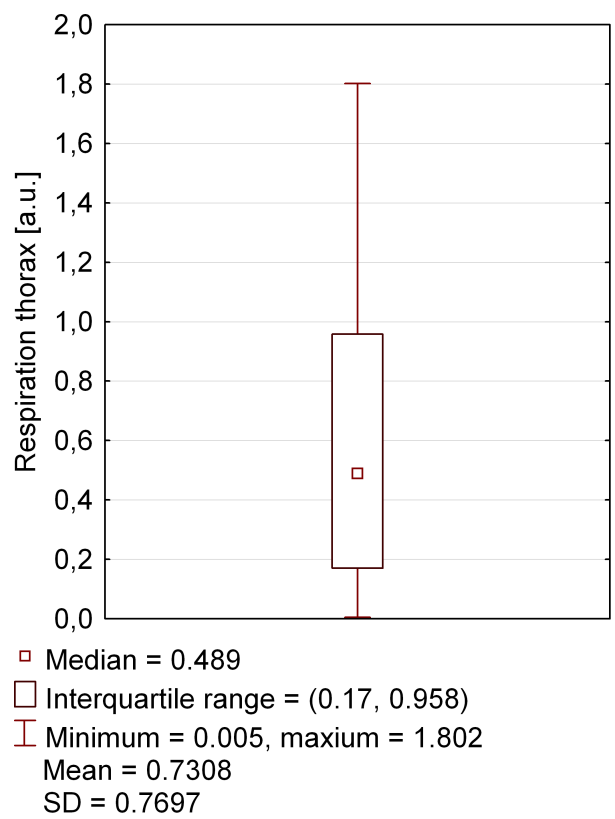

(c)

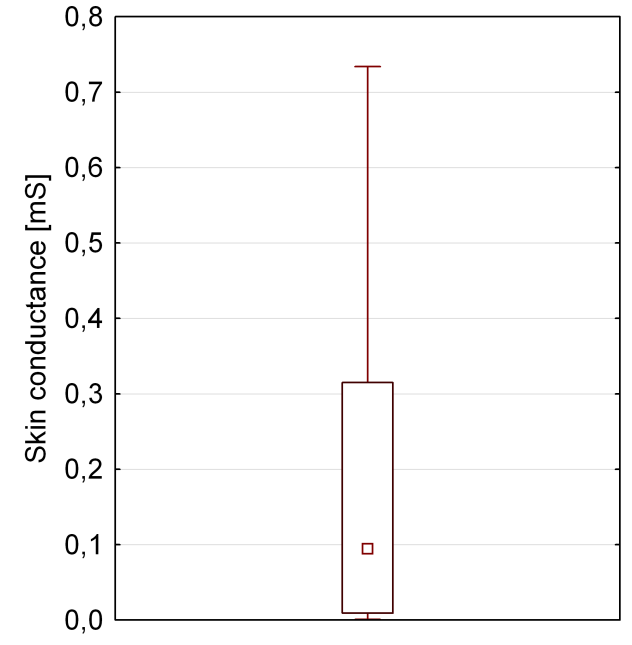

- Median $=0.0945$

$\square$ Interquartile range $=(0.009,0.315)$

I Minimum $=0.001$, maximum $=0.734$

Mean $=0.2778$

$\mathrm{SD}=0.4783$

(b)
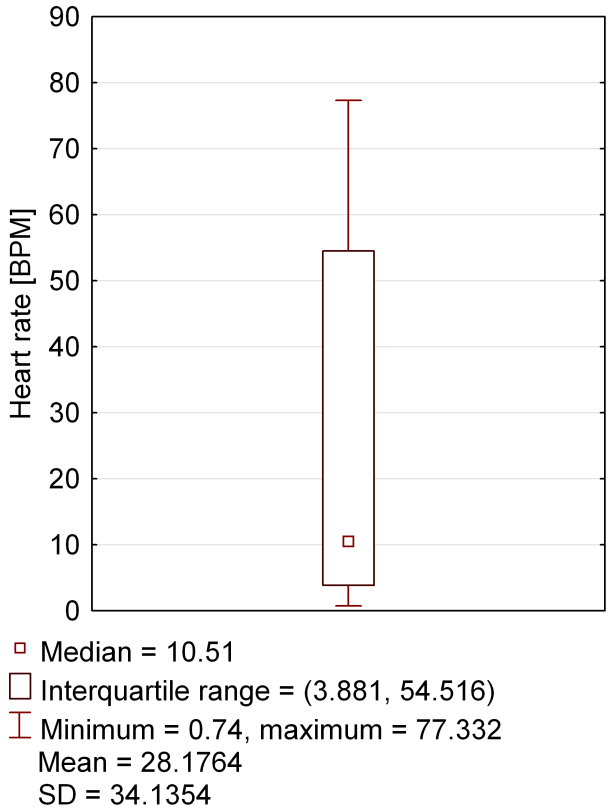

(d)

Fig. 3. Basic statistics for measured physiological parameters that show the biggest changes due to stressful condition: (a) BVP, (b) SC, (c) respiration thorax and (d) heart rate.

hyperventilation is caused by stress factors. ${ }^{19-21}$ This explains the changes in respiration of patients in response to a shot sound. Therefore, we decided that SC and respiration shall be measured in the system supporting behavioral therapy of children with autism.
On the basis of conducted study, a prototype of the system supporting behavioral therapy for children with autism was designed and realized. The system was named the "Detector MED-4".

Detector MED-4 is a microprocessor-based portable device, which enables detection and indication 


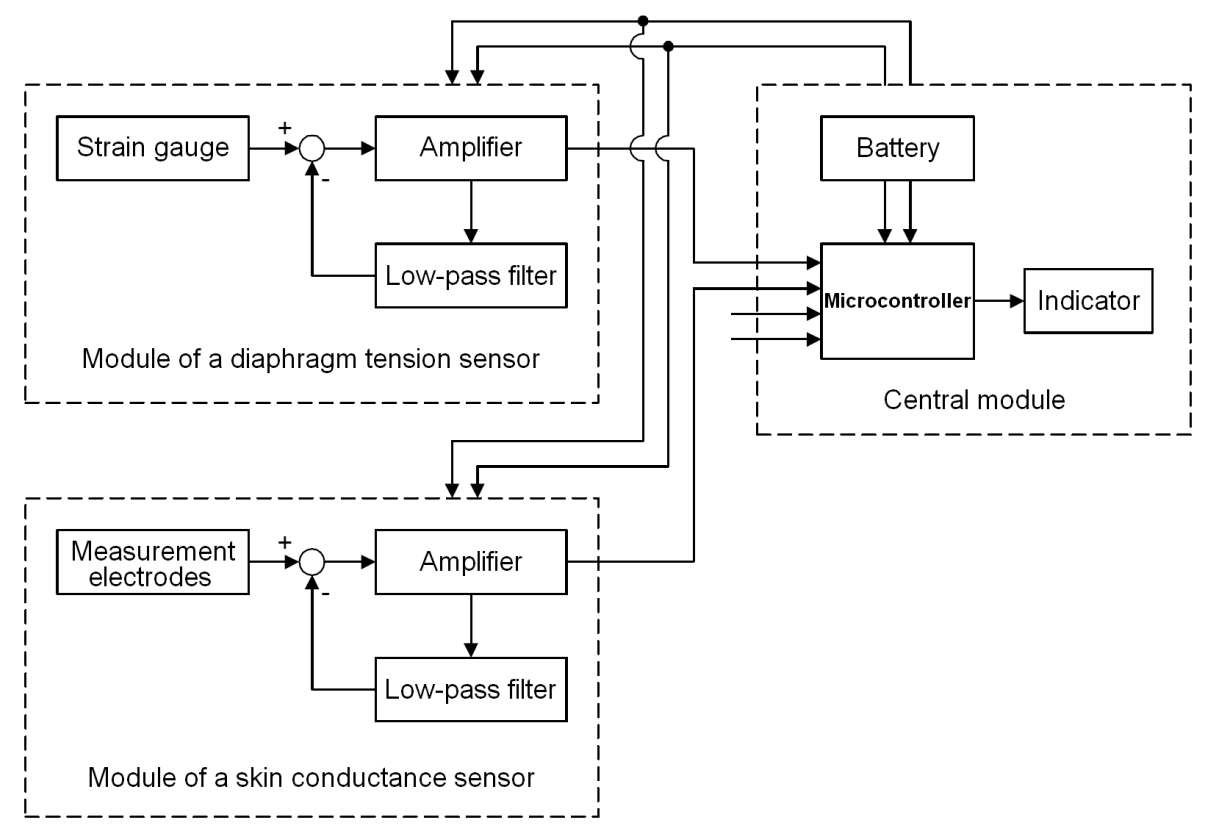

Fig. 4. Block diagram of Detector MED-4.

of changes in physical properties that occur in an individual under stress. The device consists of four modules:

- central module,

- indicator,

- module of a diaphragm tension sensor,

- module of a SC sensor.

Block diagram of the developed device is shown in Fig. 4.

Module of a SC sensor is presented in Fig. 5. This sensor is a self-calibrating device. It adapts to the person's skin resistance and to the small deviations in measured signal.

Module of a diaphragm tension sensor shown in Fig. 6 does not also require calibration. It adapts itself to the initial thrust of the fixing strip tension. The sensor does not respond to slow changes in pressure force caused by patient's breathing.

Information about the rising time and duration of the measured signals is transmitted separately from each sensor to the central module.

Central module collects information from both sensors. Changes in the values of measured signals are ignored by software if their duration lasts less than $0.2 \mathrm{~s}$. The system indicates a change in the emotional state of volunteer only if the measured signal from one sensor is alarming about a possible stress, it is confirmed by the signal from the second sensor within $3 \mathrm{~s}$. Then, the signaling module lights up in blue. Signaling lasts for $5 \mathrm{~s}$ after the cessation of the changes recorded by the sensors. Central module includes a power switch and two LEDs indicating the occurrence of changes recorded by the external sensors:

- yellow LED indicates changes detected by a SC sensor,

- green LED indicates changes detected by a diaphragm tension sensor.

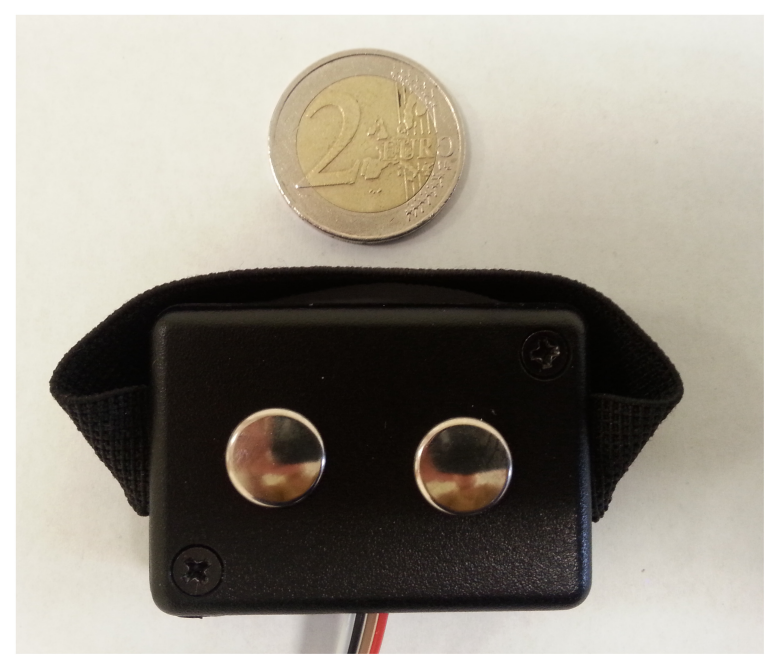

Fig. 5. Skin conductance sensor. 


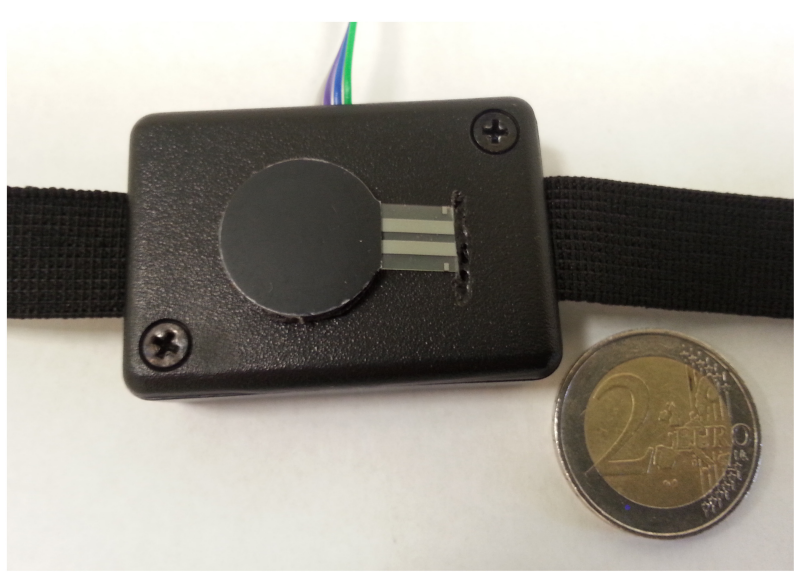

Fig. 6. Diaphragm tension sensor.

Central module and signaling module are presented in Fig. 7.

It is possible to expand the device with next two sensors. The device is powered by four AAA R03 batteries with $1.5 \mathrm{~V}$.

In order to verify the proper operation of the Detector MED-4, we conducted test with the participation of 43 volunteers. As a reference measurement method, the FlexComp Infiniti sensors were used. During the test, the object was wearing the FlexComp sensors and our systems at the same time. As a result we compare the output signal from our system and from each sensor. The test results are shown in Table 1.

The test results confirmed the proper operation of the sensor. There were 11 false-negative results; however, they were caused by sliding off the sensor strip. This will be fixed in our future work on the development of this device in a wireless version.

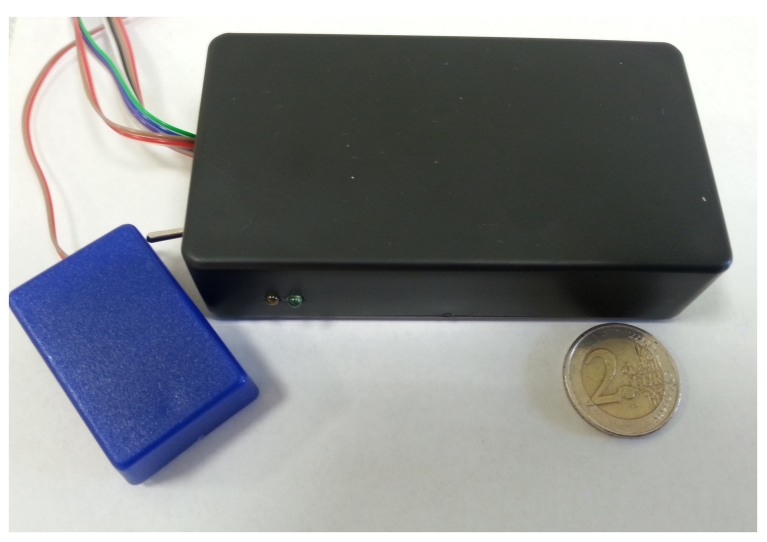

Fig. 7. Central module (black box) and signaling module (blue box).
Table 1. Results of test measurement.

\begin{tabular}{cccc}
\hline $\begin{array}{l}\text { Number of } \\
\text { reactions }\end{array}$ & $\begin{array}{c}\text { Activation of } \\
\text { the LEDs on } \\
\text { the brooch }\end{array}$ & $\begin{array}{c}\text { Change in skin } \\
\text { conductance } \\
\text { reference } \\
\text { sensor }\end{array}$ & $\begin{array}{c}\text { Change in } \\
\text { respiration - } \\
\text { reference } \\
\text { signal }\end{array}$ \\
\hline 23 & YES & YES & YES \\
2 & NO & NO & YES \\
3 & NO & YES & NO \\
4 & NO & NO & NO \\
$11^{\text {a }}$ & NO & YES & YES \\
\hline
\end{tabular}

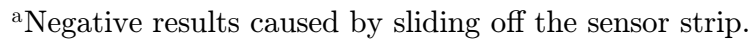

\section{Conclusion}

In this paper, a system supporting behavioral therapy for autistic children is presented. System consists of sensors network, base station and a brooch indicating person's emotional states. The selected physiological parameters were chosen during the experiment, which was designed and conducted by authors. In this experiment, a group of healthy volunteers, under controlled conditions, were exposed to a stressful situation caused by the picture or sound. The bio-statistical analysis of measured signal allowed us to discern the proper physiological parameters that are mostly associated to changes due to emotional state of a patient, such as: SC, temperatures and respiration rate. The respiration rate and skin conductance changes are chosen to be monitored in the elaborated system.

Recently, this system has been tested in one kindergarten for children with disabilities. Teachers, who tested it, pointed out that it is really hard to teach the autistic children the correlation between teacher's mood and the visual signal. However, this system seems to be a very good tool for children selflearning their own emotions, which they are normally not able to recognize. Such support will be very useful and can significantly improve psychological treatment of those children.

The investigation of these sensors confirms their ability for the respiration and skin conductance monitoring in the system supporting behavioral therapy for children with autism. In this paper, we present preliminary results which can be the basis for building sensors network ready for practical applications. After the clinical trials, which are to confirm its usefulness, the system will be rebuilt for 
a more wearable system that will consist of optoelectronic sensors ${ }^{22,23}$ and wireless communication between sensors and the central unit/base station.

\section{Acknowledgment}

This study was partially supported by the Foundation for Polish Science under grant No. 48/UD/ SKILLS/2014 and the National Centre for Research and Development, Poland under grant titled: "Automated therapy monitoring for children with developmental disorders of autism spectrum", as well as DS Programs of the Faculty of Electronics, Telecommunications and Informatics, Gdańsk University of Technology, and European Cooperation in Science and Technology (COST) Action TD1309.

\section{References}

1. E. Ikeda, E. Hinckson, Ch. Krageloh, "Assessment of quality of life in children and youth with autism spectrum disorder: A critical review," Qual. Life Res. 23, 1069-1085 (2014).

2. J. Schroeder, M. Cappadocia, J. Bebko, D. Pepler, J. Weiss, "Shedding light on a pervasive problem: A review of research on bullying experiences among children with autism spectrum disorders," J. Autism Dev. Disord. 44, 1520-1534 (2014).

3. K. Williams, S. Woolfenden, J. Roberts, S. Rodger, L. Bartak, M. Prior, "Autism in context 1: Classification, counting and causes," J. Paediatr. Child Health 50, 335-340 (2014).

4. A. Sawyer, J. Lake, Y. Lunsky, S. Liu, P. Desarkar, "Psychopharmacological treatment of challenging behaviours in adults with autism and intellectual disabilities: A systematic review," Res. Autism Spectr. Disord. 8, 803-813 (2014).

5. W. Szwoch, "Using physiological signals for emotion recognition ", Proc. HSI (2014), pp. 556-561.

6. J. Healey, R. Picard, "Detecting stress during realworld driving tasks using physiological sensors," IEEE Trans. Intell. Transp. Syst. 6(2), 156-166 (2005).

7. C. Lisetti, F. Nasoz, "Using noninvasive wearable computers to recognize human emotions from physiological signals," EURASIP J. Appl. Signal Process. 11, 1672-1687 (2004).
8. I. Mauss, M. Robinson, "Measures of emotion: A review," Cogn. Emot. 23(2), 209-237 (2009).

9. E. Jang, B. Rak, S. Kim, J. Sohn, "Emotion classification by Machine Learning Algorithm using Physiological Signals," Vol. 5, Proc. Computer Science and Information Technology (2012), pp. 1-5.

10. D. Bach, G. Flandin, K. Friston, R. Dolan, "Modelling event-related skin conductance responses," Int. J. Psychophysiol. 75(3), 349-356 (2010).

11. A. Boxtel, "Facial EMG as a tool for inferring affective states," Proc. Measuring Behavior (2010), pp. $104-108$.

12. A. Landowska, "Emotion monitor — verification of physiological characteristics measurement procedures," Metrology Measurement Syst. J. 4, (2014) to be published.

13. BioGraph Infiniti and FlexComp Infiniti User Manual, Thought Technology, Canada, www. thoughttechnology.com (VII 2013).

14. K. Karpienko, M. S. Wróbel, R. Urniaż, "Reliability and validity of optoelectronic method for biophotonical measurements," Vol. 9032, Progress in Biomedical Optics and Imaging Biophotonics - Riga 2013 Proc. of SPIE (2013), pp. 90320Q-1-90320Q-9.

15. D. A. Freedman, R. Pisani, R. Purves, Statistics, Norton \& Company Incorporated, New York (2007).

16. F. Martini, E. Bartholomew, Essentials of Anatomy Es Physiology, Benjamin Cummings, San Francisco (2003).

17. J. D. Montagu, E. M. Coles, "Mechanism and measurement of the galvanic skin response," Psychol. Bull. 65(5), 261-279 (1966).

18. R. E. Brashear, "Hyperventilation syndrome," Lung, 161(1), 257-273 (1983).

19. D. S. Cowley, P. P. Roy-Byrne, "Hyperventilation and panic disorder," Am. J. Med. 83(5), 929-937 (1987).

20. H. Folgering, "The pathophysiology of hyperventilation syndrome," Monaldi Arch. Chest Dis. 54(4), 365-372 (1999).

21. B. Garssen, "Role of stress in the development of the hyperventilation syndrome," Psychother. Psychosom. 33, 214-225 (1980).

22. A. A. Zanishevskaya, A. V. Malinin, V. V. Tuchin, Yu. S. Skibina, I. Yu. Silokhin "Photonic crystal waveguide biosensor," J. Innov. Opt. Health Sci. 6(2), 1350008 (2013).

23. M. Jedrzejewska-Szczerska, M. Gnyba, B. B. Kosmowski, "Low-coherence fibre-optic interferometric sensors," Acta Physica Polonica A 120(4), 621-624 (2011). 